\title{
Congenital spinal dermal tract: how accurate is clinical and radiological evaluation?
}

\author{
Martin M. Tisdall, MD, Richard D. Hayward, FRCS, and Dominic N. P. Thompson, FRCS \\ Department of Neurosurgery, Great Ormond Street Hospital for Children NHS Trust, London, United Kingdom
}

OBJECT A dermal sinus tract is a common form of occult spinal dysraphism. The presumed etiology relates to a focal failure of disjunction resulting in a persistent adhesion between the neural and cutaneous ectoderm. Clinical and radiological features can appear innocuous, leading to delayed diagnosis and failure to appreciate the implications or extent of the abnormality. If it is left untreated, complications can include meningitis, spinal abscess, and inclusion cyst formation. The authors present their experience in 74 pediatric cases of spinal dermal tract in an attempt to identify which clinical and radiological factors are associated with an infective presentation and to assess the reliability of MRI in evaluating this entity.

METHODS Consecutive cases of spinal dermal tract treated with resection between 1998 and 2010 were identified from the departmental surgical database. Demographics, clinical history, and radiological and operative findings were collected from the patient records. The presence or absence of active infection (abscess, meningitis) at the time of neurosurgical presentation and any history of local sinus discharge or infection was assessed. Magnetic resonance images were reviewed to evaluate the extent of the sinus tract and determine the presence of an inclusion cyst. Radiological and operative findings were compared.

RESULTS The surgical course was uncomplicated in $90 \%$ of 74 cases eligible for analysis. Magnetic resonance imaging underreported the presence of both an intradural tract (MRI 46\%, operative finding $86 \%$ ) and an intraspinal inclusion cyst (MRI 15\%, operative finding $24 \%$ ). A history of sinus discharge (OR 12.8, $p=0.0003$ ) and the intraoperative identification of intraspinal inclusion cysts (OR 5.6, $p=0.023$ ) were associated with an infective presentation. There was no significant association between the presence of an intradural tract discovered at surgery and an infective presentation.

CONCLUSIONS Surgery for the treatment of spinal dermal tract carries a low morbidity. While it seems intuitive that tracts without intradural extension carry a low risk of spinal cord tethering, it is not possible to reliably detect these cases using MRI. Similarly, intraspinal dermoid cannot be reliably excluded using MRI and carries an increased risk of infection. These points justify excision together with intradural exploration of all spinal dermal sinus tracts.

http://thejns.org/doi/abs/10.3171/2014.11.PEDS14341

KEY WORDS spinal dysraphism; spinal dermal tract; spinal dermoid; congenital; spine

$\Delta$ "DERMAL sinus tract" is defined as an abnormal tract connecting the epithelium and the spinal neural structures and containing epithelial and/or neural elements. It is part of the spectrum of occult spinal dysraphism and has an incidence of approximately 1 in 2500 live births. ${ }^{10}$ Its presumed etiology is the focal failure of disjunction resulting in an adhesion between cutaneous and neural ectoderm. ${ }^{5}$

During normal fetal development, the cutaneous ectoderm separates from the neuroectoderm in a process known as "disjunction." In the human embryo this takes place during the 4th week of development as primary neurulation and neural tube closure are completed and the neural tube becomes surrounded by the mesodermal elements that will form the vertebra, musculature, and pachymeninges.

In the context of a dermal sinus tract, an abnormal connection persists between the developing spinal cord and the integument. Thus, this tract reflects the common ectodermal origin of skin and neural tube and is typically lined with squamous epithelium. ${ }^{6}$ The tract constitutes a potential conduit for the passage of organisms and infections

ABBREVIATION VACTERL = vertebral anomalies, anal atresia, cardiac defects, tracheoesophageal fistula and/or esophageal atresia, renal and radial anomalies, and limb defects.

SUBMITTED July 1, 2014. ACCEPTED November 12, 2014.

INCLUDE WHEN CITING Published online March 13, 2015; DOI: 10.3171/2014.11.PEDS14341.

DISCLOSURE The authors report no conflict of interest concerning the materials or methods used in this study or the findings specified in this paper. 
such as meningitis or abscess may occur. ${ }^{11,12}$ Other complications such as intraspinal dermoid formation or spinal cord tethering are also recognized. ${ }^{4,7,18,19}$ Neurological impairment can result from any of these complications; thus, most surgeons currently recommend prophylactic surgery for this particular dysraphic entity. ${ }^{1,15}$

\section{Methods}

We undertook a retrospective review of all cases of dermal sinus tract presenting to a single institution over a 12-year period between 1998 and 2010. Diagnosis was based on postoperative histological findings; therefore, all patients in this analysis underwent surgery. Information regarding clinical presentation and operative findings was obtained from the clinical record and operative reports. In addition to demographic data, the nature of any cutaneous stigmata, a history of local infection or meningitis, and presenting neurological and urological status were recorded. We evaluated radiological images without knowledge of the operative findings. Axial and sagittal T1- and T2weighted images were scrutinized to assess for the presence and extent of any tract as well as any evidence of an inclusion cyst (dermoid or epidermoid). Neurological and urological status was recorded at the latest follow-up. Adverse events were identified from a prospectively maintained database of operative caseload.

Findings from preoperative MRI and intraoperative exploration were compared using 1-sample t-test with Bonferroni correction. We hypothesized that an infective presentation (subcutaneous or spinal abscess, meningitis) was associated with 3 features: an intraspinal dermoid cyst, discharge from the sinus, and an intradural tract. A 1-tailed Fisher's exact test was used to assess the strength of association between infection and these 3 factors. Bonferroni corrections were applied to account for multiple comparisons. Strengths of association are presented as odds ratios together with $95 \%$ confidence intervals, and $\mathrm{p}$ $<0.05$ was considered significant.

\section{Results}

Seventy-four children were identified for inclusion in this study. Forty (54\%) were male and 34 (46\%) were female. The mean age at surgery was 2 years 5 months (range 1 week to 16 years). Forty of the patients (54\%) were under the age of 1 year at the time of surgery.

\section{Presenting Features}

A cutaneous epithelial defect or punctum was present in 59 cases (80\%), and in 1 additional case a punctum had been excised at another institution. An area of epithelial thinning and scar-like cicatrization (cigarette burn appearance) accounted for the remaining cases (Fig. 1). Other cutaneous stigmata observed were hemangioma (28\%), hair tuft (20\%), and skin tag (8\%), with 1 additional case having had a skin tag previously excised. A subcutaneous lipoma was seen in $11 \%$ of cases. In $38 \%$ of cases, more than 1 cutaneous anomaly was present.

Prior to surgery, discharge from the sinus site was observed in 15 cases (20\%), and local infection was diagnosed in $10(14 \%)$ with 5 cases (7\%) having an episode

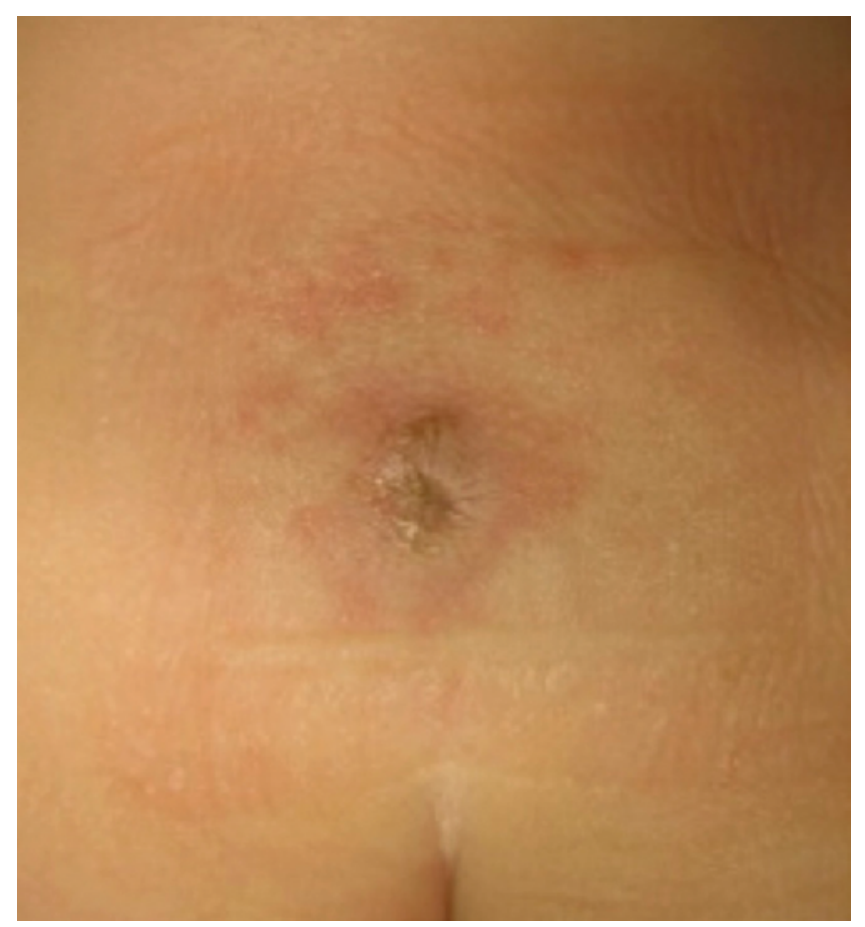

FIG. 1. Typical area of epithelial thinning and scar-like cicatrization.

of meningitis. Four cases presented with active infection. Lower-limb symptoms consisting of pain, weakness, or deformity were present in 11 cases $(15 \%)$, and back pain was present in 1 case. Bladder or bowel disturbance was present in 5 cases (7\%). Forty-three cases (58\%) were asymptomatic apart from the cutaneous stigmata.

\section{Comparison of Radiological and Intraoperative Findings}

We were able to review preoperative MRI studies for 68 of the 74 cases. A subcutaneous tract was visible on MRI in $95 \%$ of cases, and an intradural tract was visible in $46 \%$ of cases. A spinal dermoid was preoperatively identified in $15 \%$ of cases. The levels of the subcutaneous tract and the conus are shown in Figs. 2 and 3, respectively. Additional pathology was identified on MRI in 15 cases and consisted of hydromyelia (6 cases), lipoma (4 cases), fatty filum (3 cases), lipomyelomeningocele (3 cases), atretic meningocele (2 cases), split cord malformation Type II (1 case), and VACTERL association (vertebral anomalies, anal atresia, cardiac defects, tracheoesophageal fistula and/or esophageal atresia, renal and radial anomalies, and limb defects; 1 case).

\section{Surgical Technique and Findings}

The patient was prepared for surgery in a standard manner and placed prone on the operating table. Perioperative intravenous flucloxacillin and amikacin antibiotic prophylaxis was administered at induction followed by 2 postoperative doses of each agent. An elliptical incision was made around the cutaneous lesion and was deepened to the level of the fascia. Typically, a well-circumscribed defect in the fascia was found, containing the tract, which 


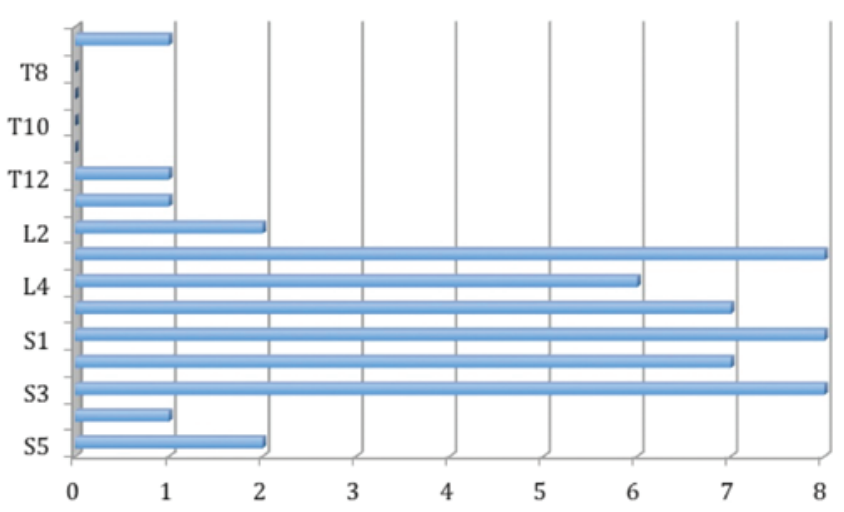

FIG. 2. Level of the spinal dermal sinus tract. Numbers on the $x$-axis represent the number of cases; the $y$-axis represents the spine levels. Figure is available in color online only.

was identified and traced through the defect in the fascia to the lamina of the vertebra. Laminoplasty was then performed in both the cranial and caudal directions in such a manner as to maintain the integrity of the tract and provide adequate access to the dura mater. Once the extradural tract was dissected, the dura was opened using an elliptical incision to include the tract, and the tract was then followed to its termination. If it was not possible to separate the tract from the spinal cord, then the tract was transected at the cord level. If an abnormally thickened filum was discovered, it was sectioned during the same procedure. The dura was closed in a watertight fashion. The residual posterior vertebral elements were reattached by fixing the laminae with 0 Vicryl sutures placed through drill holes into the bone or through the ligamentum flavum in infants and small children. Generally, a small caudal defect in the posterior spinal elements at the point of the original dysraphic abnormality remained. The wound was then closed in layers in a routine fashion.

A consistent finding at surgery was the caudal trajectory of the subcutaneous component of the tract, although after entry into the dura, the tract ascended in a cranial direction for at least 2 vertebral levels. In contrast to the MRI findings, intraoperatively an intradural tract was found in 64 cases $(86 \%)$. An intraspinal dermoid was found in 18 cases $(24 \%)$. The difference between the MRI and intraoperative findings was statistically significant $(\mathrm{p}<0.001$ for intradural tract and $p=0.028$ for intraspinal dermoid). Magnetic resonance imaging had sensitivity of 50\% and specificity of $78 \%$ for detection of the intradural tract, and sensitivity of $63 \%$ and specificity of $100 \%$ for detection of an intraspinal dermoid cyst. Examples of cases in which MRI failed to detect an intradural tract are shown in Figs. 4 and 5.

\section{Postoperative Outcome}

The postoperative course was uncomplicated in 67 cases (90\%). There was 1 case of postoperative CSF leakage and 2 cases of superficial wound infection. These 3 cases were managed conservatively.

Three cases suffered neurological deterioration relating to surgery. These cases all presented with intermittent discharge from the dermal sinus tract. Two of these cases

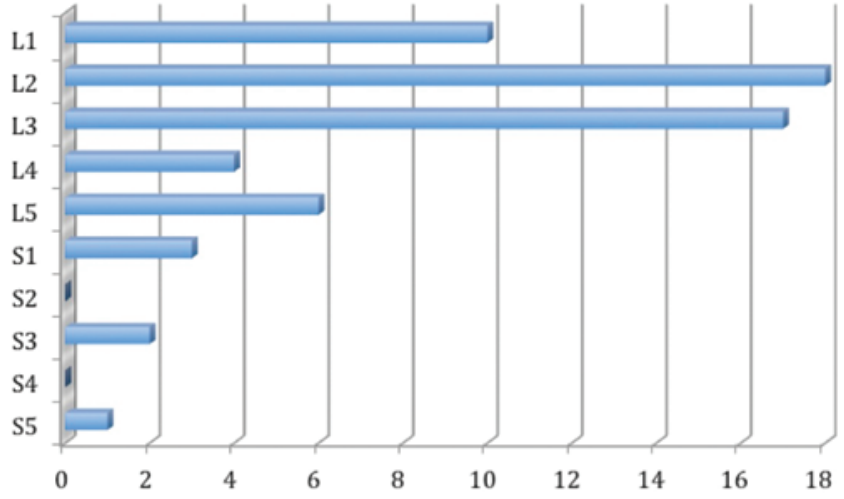

FIG. 3. Level of the conus in cases of dermal sinus tract. Numbers on the $x$-axis represent the number of cases; the $y$-axis represents the spine levels. Figure is available in color online only.

had active infection at presentation, and 1 of these 2 cases had VACTERL association and syringomyelia. Two cases involved deterioration in urinary function, 1 of which required intermittent self-catheterization, and the remaining case involved transient lower-limb weakness that completely resolved by the time of discharge from the hospital. In cases that presented without a history of discharge from the sinus tract or infection, no perioperative neurological deterioration occurred.

Two cases required a second procedure. One of these was related to wound dehiscence without infection, which was treated with debridement and wound revision. The other case initially presented with an abscess. At the first procedure it was not possible to resect the associated dermoid, and simple incision and drainage was performed, followed by antibiotic therapy. The dermoid was completely excised during a subsequent procedure.

The mean follow-up period was 37 months (range 0-149 months). Two patients had no follow-up and were therefore excluded from further analysis. By the time of the last follow-up, 65 patients (90\%) had no symptoms relating to lower-limb function, and 54 patients (75\%) had normal or age-appropriate continence. Fifty-one patients (71\%) had neither urinary nor lower-limb symptoms. Lower-limb symptoms in affected patients included pain (3 patients), unilateral weakness (1 patient), gait unsteadiness (1 patient), delayed walking (1 patient), and the need for foot splinting (1 patient). Symptoms in those without normal continence included failure to achieve fecal continence (2 cases), urinary incontinence (14 cases), neuropathic bladder dysfunction (1 case), and urinary retention (1 case). Dividing the cases according to infective presentation, we noted that $73 \%$ of cases with a history of infection were asymptomatic at the last follow-up compared with $68 \%$ of cases with no prior infection (not statistically significant).

\section{Statistical Analysis}

The presence of an intraspinal dermoid at surgery was strongly associated with an infective presentation (either meningitis or local infection) with an OR of 12.8 (95\% CI 3.5-47.1, $\mathrm{p}=0.0003$ ). Discharge from the sinus was associated with an infective presentation with an OR of 5.6 (95\% CI 1.6-19.6, $\mathrm{p}=0.023)$. There was no significant 


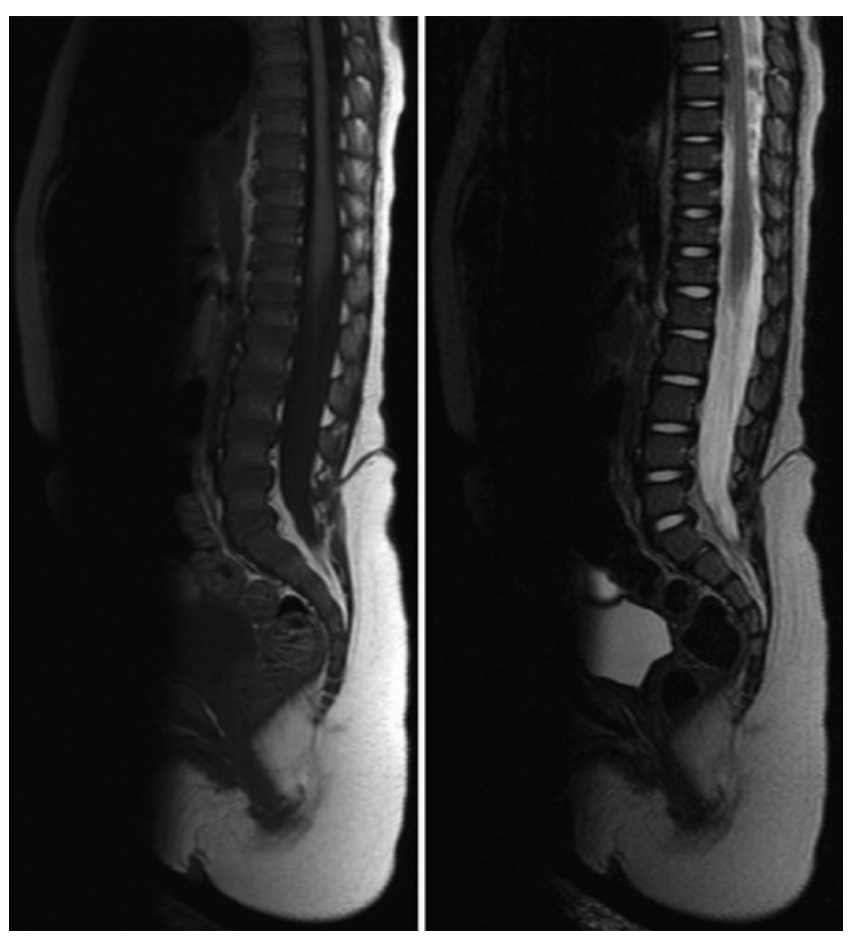

FIG. 4. Sagittal T1-weighted (left) and T2-weighted (right) MR images from a case in which the intradural tract was not identified on preoperative imaging but was found at surgery. The subcutaneous tract is clearly shown, whereas the intradural component is not visible.

association between the presence of an intradural tract, as found at surgery, and an infective presentation.

\section{Discussion}

Disjunction, the process by which the neural tube separates from the cutaneous ectoderm, has been implicated in the pathogenesis of occult spinal dysraphism. When disjunction occurs prematurely, the still-open neural tube separates from the skin and is exposed to the underlying mesoderm. Naidich et al. suggested that the differentiation of this mesoderm into fat might be the basis of lipomyelocele formation. ${ }^{13}$ In contrast, when disjunction occurs incompletely, an abnormal connection persists, in the form of a fibroneural stalk, between the skin and the spinal cord. To date, unfortunately, neither of these hypotheses has been substantiated or replicated in an animal model.

The nomenclature associated with this abnormal connection has become confused as a number of authors have sought to subdivide this entity according to factors such as clinical appearance, presumed embryogenesis, or prognostic significance. Thus, the terms "long tethering tracts" and "short tethering tracts,"16 "pseudo-dermal sinus tracts," "meningocoele manqué," myeloschisis"14 have entered the neurosurgical literature. While these may indeed be different clinicopathological entities associated with incomplete disjunction, there are particular features that they have in common and that have a bearing on clinical management and prognosis. First, neural tube closure has occurred to near or total completion; thus, significant neurological deficit is an unusual presenting feature in the absence of complications. Second, there is a physical attachment between neural and surrounding tissues with the potential for spinal cord tethering during growth. Third, the histological composition of the connecting stalk consists of a variable combination of epidermal, dermal, meningeal, and neural elements. It is perhaps the relative amounts of cutaneous versus neural tissue that is the main distinguishing feature between dermal sinus tract and limited dorsal myeloschisis. Finally, it would seem that each has the potential, albeit to a varying degree, for inclusion cyst formation and secondary infection.

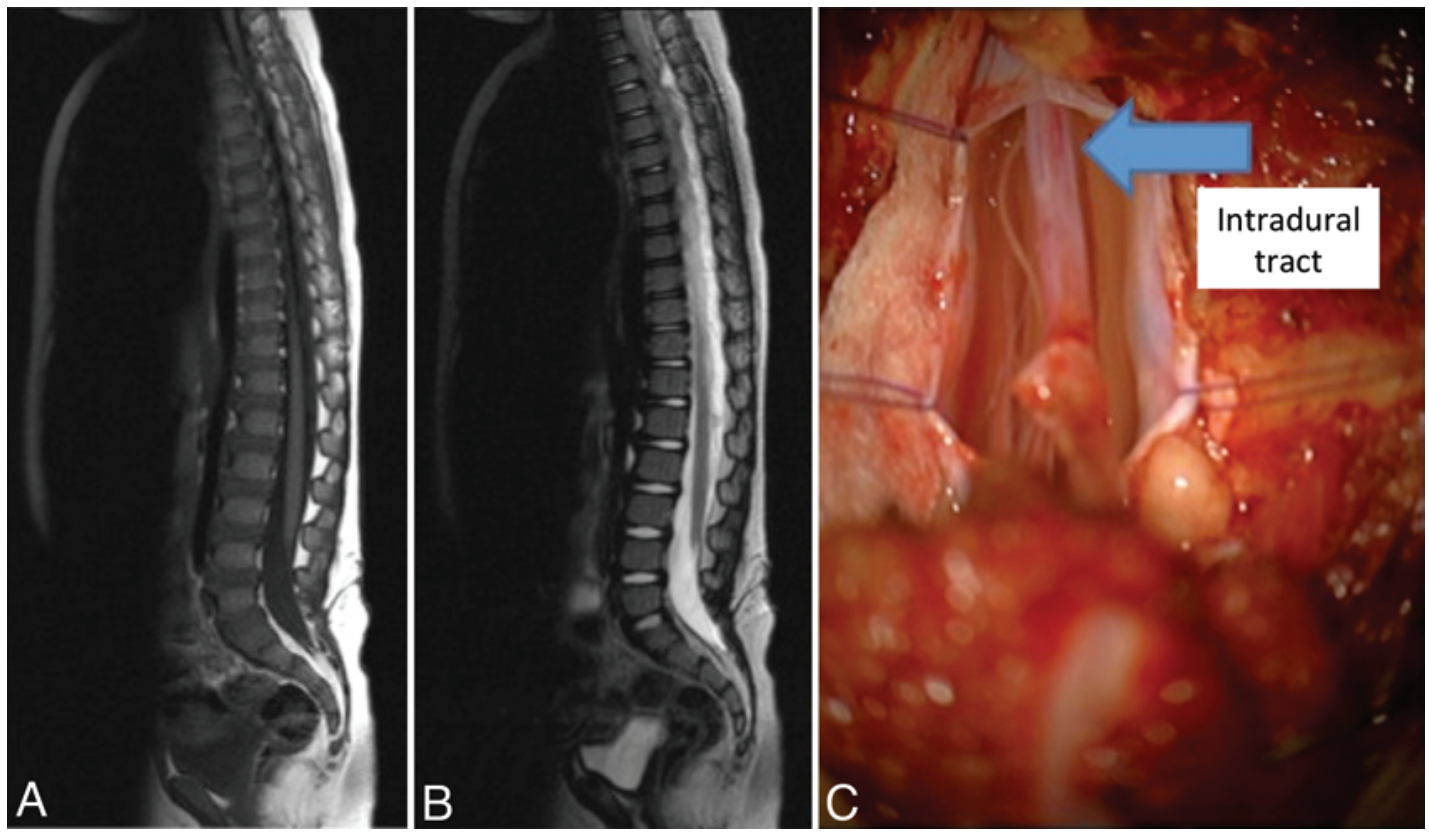

FIG. 5. Sagittal T1-weighted (A) and T2-weighted (B) MR images and intraoperative photograph (C) from a case in which the intradural tract was not identified on preoperative imaging but was found at surgery. 
Clinical management of dysraphic spinal tracts is generally determined on the basis of clinical history, examination findings, and results of MRI. While it is well recognized that infection (subcutaneous abscess, intraspinal abscess, and meningitis) can complicate a dermal sinus tract, it remains unclear what particular clinical or radiological factors predispose to this complication. The incidence of infection in a dermal sinus tract is not well characterized; however, Ackerman and Menezes found infection at presentation in $11 \%$ of cases in their series of cases collected over a 30-year period. ${ }^{1}$ Furthermore, in cases undergoing surgery, MRI may be unreliable in predicting the extent of the tract and the presence of associated pathology. ${ }^{3}$

There have been attempts to risk stratify these lesions. To date, however, it has not been possible to clearly distinguish the relative risk of complications.

In this series a clinical history of discharge from a spinal cutaneous sinus increased the likelihood of presenting with infection (OR 5.6). We identified a particularly strong association between an infective presentation and the subsequent identification of an intraspinal inclusion cyst (dermoid or epidermoid) at surgery. The need for surgical exploration in cases presenting with a history of discharge or in those known to harbor an inclusion cyst is generally undisputed and is supported by our findings.

With regard to examination findings, spinal dermal sinus tracts have been described at almost all levels of the neuraxis. Nevertheless, the majority occur in the lumbosacral region, and as disjunction plays no part in secondary neurulation, a true dermal sinus tract does not occur in the sacrococcygeal region. This feature is an aid to the clinician in distinguishing dermal sinus tracts from benign sacrococcygeal dimples or pits, which are conical depressions of the skin attached to the tip of the coccyx and are not of dysraphic significance.

Martínez-Lage et al. made a distinction between true dermal sinus tracts, which had a proven lumen and thus could act as a portal for infection, and pseudo-dermal sinus tracts, which lacked a lumen and consisted of a solid fibrovascular structure with varying amounts of neural elements. ${ }^{9}$ They suggested that this latter type was not associated with an infection risk and argued that a more cautious approach to surgery should be taken in these cases. However, their groups were selected on the basis of histological findings, both groups had patients in whom there was a skin opening, and the main clinical distinction was the presence of an open cutaneous pore (true dermal sinus tract) or a translucent membrane-covered orifice (pseudodermal sinus tract). Our experience is that the cutaneous appearance alone is not sufficiently robust as a criterion upon which to decide surgical management. In our series a histologically confirmed epithelial tract connecting the epidermis to the neural elements was present in all cases. However, a true lumen was not verified in all cases, and in some this could only be seen in part rather than along the entire length of the tract. We submit that the presence and extent of the lumen is not consistently predictable from its clinical appearance or on preoperative imaging alone, and we suggest that although the depth of the lumen may affect the risk of infection, it is extremely difficult to accurately ascertain the risk of infection prior to surgery. While we would advocate for earlier surgery in those cases in which a deep lumen is seen, particularly when there has been a history of discharge or infection, we caution against underplaying the risk of infection in cases in which only a small punctum is seen, especially given the possible presence of an occult intraspinal dermoid on MRI. Cases presenting with an intraspinal abscess constitute a neurosurgical emergency, yet the local edema and hyperemia often make complete resection of the cyst in the acute setting a complex procedure. ${ }^{17}$ We would advocate an initial surgery limited to drainage of the abscess to decompress neural structures and obtain tissue for microbiological analysis. Definitive surgery can then be performed once the acute infection has been adequately treated. ${ }^{19}$

Even in the asymptomatic child, dermal sinus tract excision is justified given that the surgical risk is low and the potential consequences of an infective complication, if left untreated, remain. We advocate surgical exploration of all lesions thought to represent spinal dermal sinus tracts. In this series 3 cases suffered neurological deterioration with one of these cases having a transient limb weakness with a subsequent full recovery. Despite an uncomplicated surgical course, several patients continued to have neurological or urological deficits at the last follow-up. A proportion of patients in this study had other associated dysraphic abnormalities, which may have contributed to their final neurological outcome. The association of a dermal sinus tract with other dysraphic anomalies is well characterized.

There is little literature concerning the reliability of MRI in the evaluation of dermal sinus tracts. Contrast MRI was not performed in this study, and this might increase the sensitivity of MRI for detection of the tract. ${ }^{2}$ It is recognized that MRI is frequently unreliable in distinguishing between dermoid and epidermoid. ${ }^{20,21}$ Furthermore, MRI may fail to detect inclusion cysts altogether. ${ }^{3}$ Our experience is that conventional MRI sequences, while showing good specificity for the presence of intraspinal inclusion cysts, are not sufficiently sensitive to exclude this possibility. Magnetic resonance imaging should not be relied on to exclude the diagnosis of an inclusion cyst when the clinical history is suggestive. One case in this series was referred from another institution after having presented with recurrent episodes of infection following surgery for a sinus infection. A cutaneous sinus had been excised, though no intradural exploration was undertaken as the MRI sequences did not reveal an intradural tract or dermoid. Subsequent intradural exploration confirmed a dermoid and a tract within the cauda equina.

It might seem intuitive that cases without an intradural tract would have a reduced risk of complications and thus that MRI might aid risk stratification. In our series, however, MRI had a sensitivity of only $50 \%$ for the detection of an intradural tract. The difficulty in identifying the intradural portion of a tract was noted as long ago as 1991 by Barkovich et al., who attributed the problem to the small diameter of the tract and the tendency for tracts to be isointense to CSF on short repetition time/echo time sequences. ${ }^{3}$ Clearly, MRI remains an essential tool in the evaluation of this subgroup of spinal dysraphism; however, it is important for clinicians to recognize that MRI has a 
false-negative rate both for the detection of inclusion cysts and, in particular, for the intradural component of the tract.

\section{Conclusions}

Cutaneous midline sinuses in children merit close scrutiny. While these cases may be etiologically heterogeneous, infection is a feared complication and can be associated with long-term neurological and urological disability. Intraspinal infectious complications of dermal sinuses are more likely when there has been a history of discharge from the sinus and in the presence of a spinal inclusion cyst. Magnetic resonance imaging cannot reliably exclude the presence of a dermoid nor confirm the intradural extent of the tract. We would advocate intradural exploration in all cases.

\section{References}

1. Ackerman LL, Menezes AH: Spinal congenital dermal sinuses: a 30-year experience. Pediatrics 112:641-647, 2003

2. Algra PR, Hageman LM: Gadopentetate dimeglumine-enhanced MR imaging of spinal dermal sinus tract. AJNR Am J Neuroradiol 12:1025-1026, 1991

3. Barkovich AJ, Edwards Ms, Cogen PH: MR evaluation of spinal dermal sinus tracts in children. AJNR Am J Neuroradiol 12:123-129, 1991

4. Elton S, Oakes WJ: Dermal sinus tracts of the spine. Neurosurg Focus 10(1):E4, 2001

5. French BN: The embryology of spinal dysraphism. Clin Neurosurg 30:295-340, 1983

6. French BN: Midline fusion defects and defects of formation, in Youmans JR (ed): Neurological Surgery, ed 3. Philadelphia: Saunders, 1990, pp 1081-1235

7. Kanev PM, Park TS: Dermoids and dermal sinus tracts of the spine. Neurosurg Clin N Am 6:359-366, 1995

8. Lassman LP, James CC: Meningocoele manqué. Childs Brain 3:1-11, 1977

9. Martínez-Lage JF, Almagro MJ, Ferri-Ñiguez B, Izura Azanza V, Serrano C, Domenech E: Spinal dermal sinus and pseudo-dermal sinus tracts: two different entities. Childs Nerv Syst 27:609-616, 2011

10. McComb JG: Congenital dermal sinus, in Pang D (ed): Disorders of the Pediatric Spine. New York: Raven Press, 1995, pp 349-360

11. Morandi X, Mercier P, Fournier HD, Brassier G: Dermal sinus and intramedullary spinal cord abscess. Report of two cases and review of the literature. Childs Nerv Syst 15:202208, 1999
12. Mount LA: Congenital dermal sinuses as a cause of meningitis, intraspinal abscess and intracranial abscess. J Am Med Assoc 139:1263-1268, 1949

13. Naidich TP, McLone DG, Mutluer S: A new understanding of dorsal dysraphism with lipoma (lipomyeloschisis): radiologic evaluation and surgical correction. AJR Am J Roentgenol 140:1065-1078, 1983

14. Pang D, Zovickian J, Oviedo A, Moes GS: Limited dorsal myeloschisis: a distinctive clinicopathological entity. Neurosurgery 67:1555-1580, 2010

15. Radmanesh F, Nejat F, El Khashab M: Dermal sinus tract of the spine. Childs Nerv Syst 26:349-357, 2010

16. Rajpal S, Salamat MS, Tubbs RS, Kelly DR, Oakes WJ, Iskandar BJ: Tethering tracts in spina bifida occulta: revisiting an established nomenclature. J Neurosurg Spine 7:315-322, 2007

17. Sandler AL, Thompson D, Goodrich JT, van Aalst J, Kolatch E, El Khashab M, et al: Infections of the spinal subdural space in children: a series of 11 contemporary cases and review of all published reports. A multinational collaborative effort. Childs Nerv Syst 29:105-117, 2013

18. Swift DM, Carmel PW: Congenital intradural pathology. Neurosurg Clin N Am 1:551-567, 1990

19. Thompson DNP: Spinal inclusion cysts. Childs Nerv Syst 29:1647-1655, 2013

20. van Aalst J, Hoekstra F, Beuls EAM, Cornips EMJ, Weber JW, Sival DA, et al: Intraspinal dermoid and epidermoid tumors: report of 18 cases and reappraisal of the literature. Pediatr Neurosurg 45:281-290, 2009

21. Vion-Dury J, Vincentelli F, Jiddane M, Van Bunnen Y, Rumeau C, Grisoli F, et al: MR imaging of epidermoid cysts. Neuroradiology 29:333-338, 1987

\section{Author Contributions}

Conception and design: Hayward, Thompson. Acquisition of data: Tisdall. Analysis and interpretation of data: Tisdall. Drafting the article: Tisdall. Critically revising the article: all authors. Reviewed submitted version of manuscript: all authors. Approved the final version of the manuscript on behalf of all authors: Tisdall. Statistical analysis: Tisdall.

\section{Correspondence}

Martin Tisdall, Department of Paediatric Neurosurgery, Great Ormond Street Hospital for Children NHS Trust, Great Ormond St., London WC1N 3JH, United Kingdom. email: mtisdall@ me.com. 